\title{
The influence of silver nanoparticles on chicken embryo development and bursa of Fabricius morphology
}

\author{
M. Grodzik ${ }^{1}$ and E. Sawosz \\ Warsaw Agricultural University, \\ Department of Animal Nutrition and Feed Science \\ Ciszewskiego 8, 02-786 Warsaw, Poland
}

\begin{abstract}
The aim of the experiment was to determine the influence of colloidal silver nanoparticles on the development of chicken embryos, particularly on the bursa of Fabricius. Silver nanoparticles $(10 \mathrm{ppm})$ were injected into fertilized chicken eggs on days 5, 11 and 17. Ag nanoparticles did not influence embryo development as evaluated by the Hamilton and Hamburger standard. They did, however, decrease lymph follicular number and size, and changed the profile of the cell nuclei in the bursa of Fabricius.
\end{abstract}

KEY WORDS: chicken, embryo, silver, nanoparticles, bursa of Fabricius, morphology

\section{INTRODUCTION}

Silver is known for its medicinal properties, especially as an anti-microbial agent, but it may be toxic when it is in ionic state. However, the toxicity of Ag can be eliminated, when used in nanoparticle form. Nanoparticles are structures from 1 to $100 \mathrm{~nm}$. Due to their small size, the total surface area of the particles exposed in solution is maximized, resulting in the highest activity per unit of weight. In our earlier in vitro experiments, colloidal silver nanoparticles (5 ppm) showed strong antimicrobial properties against Salmonella enteritidis and Escherichia coli (Lepianka et al., unpublished). We hypothesized that silver nanoparticles (nano-Ag) used as an antimicrobial agent can stimulate development as well as immunity.

The aim of the present experiment was to evaluate the influence of colloidal $\mathrm{Ag}$ nanoparticles on the development status of chicken embryos, particularly on the bursa of Fabricius (BF).

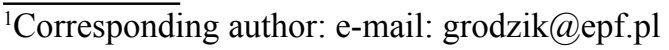




\section{MATERIAL AND METHODS}

Ninety fertile eggs (54-58 g) from Ross hens were incubated at $37.7^{\circ} \mathrm{C}, 60 \%$ humidity and automatically turned every hour. Eggs were divided into three groups (control, placebo and nano-Ag) and injected 3 times during incubation on days 5, 11 and 17; the placebo group, with $0.2 \mathrm{ml} 0.9 \% \mathrm{NaCl}$, the nano-Ag group, with $0.2 \mathrm{ml} 10 \mathrm{ppm}$ colloidal $\mathrm{Ag}$ nanoparticles. On day 18 of incubation the eggs were weighed, opened and the embryos sacrificed by decapitation. The embryos' livers, hearts and eyes were weighed and examined according to the Hamburger and Hamilton standard (1951).

Bursae of Fabricius were collected and fixed in 4\% paraformaldehyde in phosphate-buffered saline(PBS; Sigma-Aldrich) overnight at $4{ }^{\circ} \mathrm{C}$ and in a methanol gradient (Merck). Cryostat sections $(10 \mu \mathrm{m}$ thick) were air dried on slides with poly-L-lysine (Menzel GmbH Co.KG). Air-dried sections were rehydrated in PBS. Both sections were incubated with $0.02 \mu \mathrm{g} \cdot \mathrm{ml}^{-1} 4^{\prime}$.6-diamidino-2-phenyl-indole (DAPI) in PBS/T (0.5\% Tween 20 in PBS) for 20 min and washed three times with PBS (5 min $\times 3$ ). All cover slips were mounted on slides with Fluoromount (SBA). Stained specimens were observed using an Olympus FV1000 microscope.

A concentrate $(50 \mathrm{ppm})$ of colloidal silver nanoparticles was obtained from Nano-tech (Poland). The solution was diluted to concentration of $10 \mathrm{ppm}$ by adding $0.9 \% \mathrm{NaCl}$. The results were analysed by one-way ANOVA using the Statgraphics 4.1 Plus software package.

\section{RESULTS AND DISCUSSION}

The injected colloidal silver nanoparticles $(3 \times 10 \mathrm{ppm})$ did not influence the development of chicken embryos. Mortality and number of abnormalities were similar in all groups. The weight of embryos as well as the relative weights of the liver, heart and eye did not differ among the nano-Ag, control, and placebo groups (Table 1). Administration of nano-Ag had no effect on embryo morphology. Nanoparticles of Ag interact with microorganisms via preferential binding to the exposed sulphur-bearing residues of the glycoprotein knobs (Elechiguerra et al., 2005 ) without entering into animal cells and probably therefore had no detrimental effects on embryos.

The structure of BF (lymph follicle numbers and size, 100x) and profile of the cell nuclei $(1000 \mathrm{x})$ were viewed by confocal microscopy (Figure 1). The oval bursa contained primary canals located between follicles. Blind, branched secondary canals extended from the primary structures. The surface of these canals in the nano-Ag group was larger and more wrinkled than in the control and placebo groups, probably due to the reduced number and size of follicles. 
Table 1. Weight of eggs, embryos, and selected organs

\begin{tabular}{lrrrrrr}
\hline \multirow{2}{*}{ Item } & \multicolumn{3}{c}{ Treatment } & & \multicolumn{2}{c}{ ANOVA } \\
\cline { 2 - 3 } \cline { 6 - 7 } & control & placebo & nano-Ag & & SEM & P \\
\hline Egg weight, g & 54.53 & 55.03 & 55.13 & & 1.697 & 0.19 \\
Embryo weight, g & 27.70 & 27.57 & 28.26 & & 0.439 & 0.63 \\
Liver, g/100 g body mass & 2.40 & 2.59 & 2.55 & & 0.088 & 0.40 \\
Heart, g/100 g body mass & 0.98 & 1.05 & 0.98 & & 0.035 & 0.43 \\
Eye, g/100 g body mass & 1.71 & 1.84 & 1.77 & & 0.054 & 0.40 \\
\hline
\end{tabular}
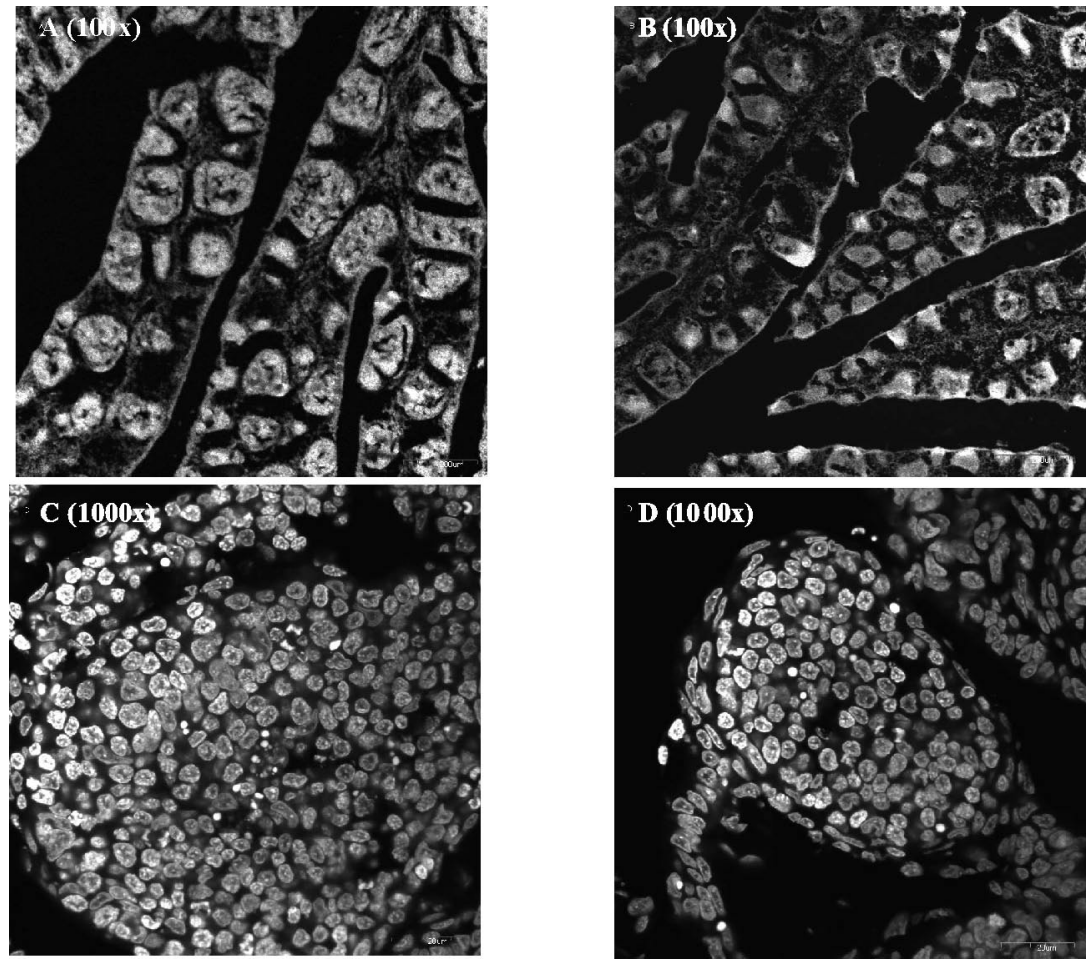

Figure 1. DAPI staining of BF from day 18 chicken embryo (A, C - control; B, D - nano-Ag)

The number and luminal intensity of cell nuclei was also decreased in the BF of the nano-Ag group. However, the size and shape of nuclei and the chromatin arrangement in most nuclei did not change. Furthermore, histological examination failed to reveal differences (control/placebo vs nano-Ag) in the occurrence of apoptotic signs such as chromatin condensation, nuclear fragmentation, and formation of apoptotic bodies. Moreover, there were no signs of necrosis. We observed a slight increase in the population of cells with peripherally deeply staining nuclei in the nano-Ag group, but more advanced methods have to be used in order to identify these cells. 
Nevertheless, the morphological picture of the bursa may indicate that Ag rather inhibited the development of the bursa than degraded an already developed organ.

The $\mathrm{BF}$ is the primary site of $\mathrm{B}$ lymphocyte immigration and early proliferation. However, the first wave of immigrated blood-borne cells (on day 6.5) are bursal dendritic cells, which produce and secrete "bursa-specific substances" inside the epithelium (Nagy et al., 2004). These substances contribute to the bursal microenvironment and B-lymphocyte maturation, and play a key role in development of BF. According to Moore et al. (2004) an ovoinhibitor is produced in the bursal dendritic-like cells, found at the cortico-medullary border of the BF. Hypothetically, these ovoinhibitors exhibit a cell signaling activity, probably for B-cell expansion and gene conversion, showing some structural homology to IL-1 (Moore et al., 2004). Other investigations demonstrated that an anti-bursin monoclonal antibody decreased lymphocyte proliferation, development of follicles and BF weight (Guo et al., 2006).

Injection of $\mathrm{Ag}$ nanoparticles on the day 5 could influence colonization or/and further secretion of bursal secretory dendritic-like cells within the BF. Data concerning the bioactivity of silver nanoparticles in tissues is scanty, but an influence of $\mathrm{Ag}$ nanoparticles on cytokine production was observed by Bhol and Schechter (2005).

\section{CONCLUSIONS}

Colloidal silver nanoparticles (10 ppm) injected into fertilized eggs on days 5, 11 and 17 of incubation did not influence the development of embryos, but decreased the number and size of lymph follicles in the bursa of Fabricius.

\section{REFERENCES}

Bhol K.C., Schechter P.J., 2005. Topical nanocrystaline silver cream suppresses inflammatory cytokines and induces apoptosis of inflammatory cells in a murine model of allergic contact dermatitis. Brit. J. Dermatol. 6, 1235-1242

Elechiguerra J.L., Burt J.L., Morones J.R., Camacho-Bragado A., Gao X., Lara H.H., Yacaman M.J., 2005. Interaction of silver nanoparticles with HIV-1. J. Nanobiotechnol. 3, 6-11

Guo S., Chen N.H., Guan R., Feng J., Huang W., 2006. Effect of anti-bursin monoclonal antibody on immunosuppresion in the duck (Cherry Valley duck). Poultry Sci. 85, 258-265

Hamburger V., Hamilton H.L., 1951. A series of normal stages in the development of the chick embryo. Develop. Dynam. 195, 231-72

Moore R.W., Hargis B.M., Porter E.T., Caldwell D.Y., Oubre C.M., Vandesande F., Berghman L.R., 2004. Ovoinhibitor in the chicken bursa of Fabricius: Identification, isolation and localisation. Cell Tissue Res. 317, 247-251

Nagy N., Magyar A., Toth M., Olah I., 2004. Origin of the bursal secretory dendritic cell. Anat. Embryol. 208, 97-107 